\title{
Additive Manufacturing of Composites and Complex Materials
}

\author{
JONATHAN E. SPOWART,${ }^{1}$ NIKHIL GUPTA $®{ }^{2,5}$ and DIRK LEHMHUS ${ }^{3,4}$ \\ 1.-Air Force Research Laboratory, Materials and Manufacturing Directorate Wright-Patterson \\ AFB, Dayton, OH 45433, USA. 2.-Composite Materials and Mechanics Laboratory, Mechanical \\ and Aerospace Engineering Department, Tandon School of Engineering, New York University, \\ Brooklyn, NY 11201, USA. 3.-Fraunhofer Institute for Manufacturing Technology and Advanced \\ Materials (IFAM), 28359 Bremen, Germany. 4.-MAPEX Center for Materials and Processes, \\ University of Bremen, 28359 Bremen, Germany. 5.-e-mail: ngupta@nyu.edu
}

Advanced composite materials form an important class of high-performance industrial materials used in weight-sensitive applications such as aerospace structures, automotive structures and sports equipment. In many of these applications, parts are made in small production runs, are highly customized and involve long process development times. Developments in additive manufacturing (AM) methods have helped in overcoming many of these limitations. The special topic of Additive Manufacturing of Composites and Complex Materials captures the state of the art in this area by collecting nine papers that present much novel advancement in this field. The studies under this topic show advancement in the area of AM of carbon fiber and graphenereinforced composites with high thermal and electrical conductivities, development of new hollow glass particle-filled syntactic foam filaments for printing lightweight structures and integration of sensors or actuators during AM of metallic parts. Some of the studies are focused on process optimization or modification to increase the manufacturing speed or tuning manufacturing techniques to enable AM of new materials.

\section{INTRODUCTION}

Many challenges have been faced by the manufacturing industry over the years. While industry has made advancement in increasing the production speed and reducing the manufacturing cost, conventional manufacturing methods have not been able to address issues such as production of a small number of parts at low cost, production of customized or one-of-a-kind parts and production of parts at the location of their use. In addition, there are numerous examples where the desired structures cannot be manufactured by any of the conventional manufacturing methods such as casting, forging or machining. For example, designs of heat exchangers can be optimized through computational fluid dynamic analysis, but the optimized design of complex network of internal channels cannot be manufactured in one piece by traditional methods.

Jonathan Spowart, Nikhil Gupta, and Dirk Lehmhus are the guest editors for the topic Additive Manufacturing of Composites and Complex Materials in this issue. The topic is sponsored by the Composite Materials Committee of the Structural Materials Division.
Assembly or joining of numerous parts can result in issues such as design complexity, over-precise tolerancing, stress concentrations at the joints and the possibility of leakage and/or thermal fatigue. Numerous other examples can be found in the industry where limitations of conventional manufacturing methods have resulted in performance or design compromises.

In this context, advancement in the additive manufacturing (AM) methods has been very exciting because these methods can overcome many of the limitations of the conventional manufacturing methods. AM, also commonly referred to as threedimensional (3D) printing, relies upon manufacturing a component layer by layer in three dimensions from a computer-aided design (CAD) solid model. Such a scheme allows the creation of parts with internal structures, customization of each part and the manufacturing of parts in small production runs at low cost. The unique set of capabilities has made AM one of the fastest growing sectors of industry in recent years. The annual growth rate of this sector has been over $26 \%$ in the past two years, reaching 
the present value of about $\$ 6$ billion. ${ }^{1}$ The AM field is expected to grow into a $\$ 20$ billion market by the year 2020 as per some recent estimates. ${ }^{1-4}$

The intense industrial interest in the various AM methods is matched by a diverse range of activities in associated applied and basic research areas. Development of improved 3D printers with faster printing speed, developing new feed materials customized for the needs of the industry and understanding the microstructure-property correlations in AM parts are among the focus areas in applied and basic research, respectively. As the range of materials specifically developed for AM continues to grow rapidly, interest is also growing in developing AM methods capable of printing parts of various different composite materials. Advanced composite materials form an important class of highly engineered industrial materials at present. No longer considered "boutique" materials, composites have found increasing numbers of applications in aerospace structures, automobile bodies, sports equipment and consumer goods and have become an integral part of the larger set of engineering materials available to designers today, often displacing other more traditional monolithic materials because of their superior balance of properties. To capture the state of the art in this area, the symposium "Additive Manufacturing of Composites and Complex Materials II" was organized as a part of the Materials Science and Technology 2017 (MS\&T'17) conference held from 9-12 October 2017 in Pittsburgh, PA. MS\&T is organized in a joint partnership between five leading materials science-related societies-ACerS, AIST, ASM International, MetSoc and TMS. The presentations included in the symposium form the basis of organizing the present topic in JOM. However, the included papers are not limited to those presented in the symposium. Rather, a broader collection is presented to capture the state of the art in the rapidly growing area of 3D printing of composites and complex materials. Dr. Jonathan Spowart of the US Air Force Research Laboratory has served as the lead topic editor for this collection, with Drs. Gupta and Lehmhus serving as co-editors.

\section{ARTICLES INCLUDED UNDER THE TOPIC ADDITIVE MANUFACTURING OF COMPOSITES AND COMPLEX MATERIALS}

The state of the art in the present capabilities of some of the AM methods in manufacturing architectured multi-material structures and composite structures is presented in "An Overview on Additive Manufacturing of Polymers" by I. Jasiuk et al. A rapidly growing list of applications is included in the article, along with technical challenges faced by some of the AM methods. For example, the removal of excess powders from laser sintering methods, which can be difficult for many designs having enclosed areas or narrow openings, is highlighted. It is apparent from the article that advancement in manufacturing methods needs to be augmented by development of new design tools and methods to achieve the desired outcomes.

The slow manufacturing speed of most AM methods has also been a major limitation. The article "HighSpeed Additive Manufacturing through High Aspect Ratio Nozzles" by L. Shaw et al. is focused on studying the possibility of increasing the speed of AM. Increasing the deposition rate by high aspect ratio (HAR) nozzles can increase the manufacturing speed but brings many challenges such as greater flow of material reducing the dimensional control and increasing the probability of entrapping voids. The work focuses on optimizing the manufacturing parameters to obtain high-quality printed parts, while simultaneously increasing the manufacturing rate.

Functional properties such as electrical or thermal conductivity, coupled with mechanical properties, are the focus of the next two papers. The first study, "Electrical and Mechanical Properties of 3D-Printed Graphene-Reinforced Epoxy" by B. Compton et al., describes preparation of graphene dispersed epoxy inks for direct write (DW) 3D printing technology. Electrical conductivity of the nanocomposite ink specimens is much higher than the neat epoxy, while the flexural mechanical performance is identical. These properties show promise for the use of such materials and processes in electronic applications. The second study "Fiber Orientation Effects in Fused Filament Fabrication of Air-Cooled Heat Exchangers" by T. Mulholland et al. reports 3D printing of short carbon fiber-reinforced polyamide filaments. The presence of carbon fibers makes the material thermally conducting along the filament axis, but the conductivity in the normal direction is not as high because of strong anisotropy. Similar properties are witnessed in the 3D printed part, which is built by depositing the filament in closely spaced lines, where higher conductivity is measured along the printing direction compared to the normal direction. The authors conclude that toolpath planning will be at least as important as actual part geometry for AM-produced heat exchangers.

A set of two papers, "Additive Manufacturing of Syntactic Foams: Part 1: Development, Properties, and Recycling Potential of Filaments" and "Part 2: Specimen Printing and Mechanical Property Characterization" by A.K. Singh et al., presents the development of a lightweight hollow particle-filled syntactic foam filament and the optimization of 3D printing parameters of a fused filament fabrication printing method using these filaments. Syntactic foams are used in marine structures for buoyancy and other properties. AM capabilities will allow development of components that are currently not made of syntactic foams because of challenges related to failure in bonded or joined sections of syntactic foams in deep water environments.

Integration of sensors in AM parts is the focus of the next two papers. The first paper, "Integrating Fiber Optic Strain Sensors into Metal Using Ultrasonic 
Additive Manufacturing" by A. Hehr et al., uses an ultrasonic AM method capable of embedding sensors in metallic components. The low temperature of this method can enable the embedding of fiber optic sensors without damaging them. The embedded fiber Bragg grating sensors are able to measure strains under tensile and cyclic loading conditions. The second paper, "Structural Integration of Sensors/Actuators by Laser Beam Melting for Tailored Smart Components" by T. Töppel et al., reports the integration of a combined sensor and vibration actuator in a biomedical implant for promoting cell stimulation for healing (in actuation mode) and also for structural health monitoring (in sensing mode). AM allows embedding of the wireless sensor/actuator inside the implant without any joining or assembly requirements. Sensor/actuator integrated components have a wide range of applications, for example, the methods developed in this work could also have benefits for aerospace or automotive structures.

Finally, although laser beam melting (LBM) is a well-developed AM technique for metals, the poor absorptivity of oxide ceramics in near infrared (NIR) wavelengths makes it challenging to use the LBM method for ceramics. This challenge is overcome by the addition of NIR absorbers to ceramic powders in the study "Laser Beam Melting of Alumina: Effect of Absorber Additions" by L. Moniz et al. Absorbers have additional effects on powder bed densities and geometrical stability of melted tracks, both of which are studied, modeled and optimized in this paper to enable use of LBM specifically for alumina and possibly for other ceramics as well.

\section{SUMMARY}

Many exciting advances are reported in the articles included under the Additive Manufacturing of Composites and Complex Materials topic. These articles show the breadth of research activities occurring in the field of AM beyond structural metals. Development of new material systems for AM, improvement in the AM methods and sensor integration into components during AM are among the advancement reported here. It is clear that the successful development of these new manufacturing capabilities will enable novel functionalities and performance standards in components manufactured by AM and therefore this field is expected to grow at an even faster rate in coming years. As a consequence, the present topic editors will continue the series of events with a symposium on "Additive Manufacturing of Composites and Complex Materials III" at MS\&T'18 in Columbus, OH, 14-18 October 2018, and will be joined by an additional organizer, Dr. E. Jaegle of MaxPlanck-Institut für Eisenforschung (MPIE) GmbH.

The following papers are published under the topic "Additive Manufacturing of Composites and Complex Materials" in the March 2018 issue (vol. 70, no. 3) of $J O M$ and can be accessed via the $J O M$ page at http://link.springer.com/journal/11837/70/3/page/1.
- "An Overview on Additive Manufacturing of Polymers" by Iwona Jasiuk, Siyuan Pang, Christopher Kozuch, Diab Abueidda, Frances $\mathrm{Su}$, and Joanna McKittrick.

- "High-Speed Additive Manufacturing through High Aspect Ratio Nozzles" by Leon Shaw, Mashfiqul Islam, Jie Li, Ling Li, and S.M. Imran Ayub.

- "Electrical and Mechanical Properties of 3DPrinted Graphene-Reinforced Epoxy" by Brett G. Compton, Nadim S. Hmeidat, Robert C. Pack, Maximilian Heres, and Joshua R. Sangoro.

- "Fiber Orientation Effects in Fused Filament Fabrication of Air-Cooled Heat Exchangers" by T. Mulholland, S. Goris, J. Boxleitner, T.A. Osswald, and N. Rudolph.

- "Additive Manufacturing of Syntactic Foams: Part 1: Development, Properties, and Recycling Potential of Filaments" by Ashish Kumar Singh, Balu Patil, Niklas Hoffmann, Brooks Saltonstall, Mrityunjay Doddamani, and Nikhil Gupta.

- "Additive Manufacturing of Syntactic Foams: Part 2: Specimen Printing and Mechanical Property Characterization" by Ashish Kumar Singh, Brooks Saltonstall, Balu Patil, Niklas Hoffmann, Mrityunjay Doddamani, and Nikhil Gupta.

- "Integrating Fiber Optic Strain Sensors into Metal Using Ultrasonic Additive Manufacturing" by Adam Hehr, Mark Norfolk, Justin Wenning, John Sheridan, Paul Leser, Patrick Leser, and John A. Newman.

- "Structural Integration of Sensors/Actuators by Laser Beam Melting for Tailored Smart Components" by Thomas Töppel, Holger Lausch, Michael Brand, Eric Hensel, Michael Arnold, and Christian Rotsch.

- "Laser Beam Melting of Alumina: Effect of Absorber Additions" by Liliana Moniz, Christophe Colin, Jean-Dominique Bartout, Karim Terki, and Marie-Hélène Berger.

\section{ACKNOWLEDGEMENTS}

Funding support from the DAAD German Academic Exchange Service fellowship (Funding Code P 91583818) to Dr. Dirk Lehmhus to visit New York University to help plan the symposium and publication activities in Summer 2015 is acknowledged.

\section{REFERENCES}

1. T.T. Wohlers, Wohlers Report 2016: $3 D$ Printing and Additive Manufacturing State of the Industry (Wohlers Associates, 2016).

2. N. Guo and M.C. Leu, Front. Mech. Eng. 8, 215 (2013).

3. F.P. Melchels, M.A. Domingos, T.J. Klein, J. Malda, P.J. Bartolo, and D.W. Hutmacher, Prog. Polym. Sci. 37, 1079 (2012).

4. A. Takaichi, T. Nakamoto, N. Joko, N. Nomura, Y. Tsutsumi, S. Migita, H. Doi, S. Kurosu, A. Chiba, and N. Wakabayashi, J. Mech. Behav. Biomed. Mater. 21, 67 (2013). 